\title{
SUL MOTO STAZIONARIO LENTO DI UNA SFERA IN UN LIQUIDO VISCOSO.
}

\author{
Nota di Tommaso Boggio (Torino).
}

Adunanza del 12 dicembre I909.

In questa Nota mi occupo della risoluzione di alcune questioni relative al moto stazionario lento di liquidi viscosi.

Espongo dapprima le equazioni generali del problema e alcune formole per il calcolo della resistenza (e della coppia risultante), esercitata dal liquido sul corpo mobile. Quindi passo alla integrazione delle equazioni del moto stazionario lento per il caso di una sfera, considerando i due problemi interno ed esterno. I valori della velocitd e della pressione risultano espressi, mediante integrali definiti, da formole abbastanza semplici, le quali si riducono ad una forma semplicissima se le forze di massa derivano da un potenziale. Il problema interno è pure stato trattato recentemente da A. KorN ${ }^{2}$ ), risolvendolo mediante serie di funzioni sferiche; peró, col suo metodo occorrono, per i valori della velocità data in superficie, delle condizioni assai più restrittive di quelle che risultano essere sufficienti per la validità delle mie formole.

Dai risultati che ottengo, si deducono immediatamente alcune interessanti formole, relative a particolari movimenti della sfera, stabilite gid dallo STOKEs ${ }^{2}$ ).

Per ultimo determino il moto stazionario lento provocato in un liquido indefinito da un piano (indefinito) mobile in esso; le relative formole si possono dedurre da quelle stabilite nel caso della sfera, ricorrendo ad un passaggio al limite.

In questo lavoro adopero sistematicamente il metodo vettoriale, il quale, operando direttamente sugli enti geometrici (punto e vettore) che si presentano nella questione che studio, libera completamente la trattazione da ogni sorta di coordinate estranee ad essa, e inoltre conferisce molta concisione e agilità ai calcoli e alle formole ${ }^{3}$ ).

I) Allgemeine Lösung des Problems kleiner, stationärer Bewegungen in reibenden Flüssigkeiten [Rendiconti del Circolo Matematico di Palermo, t. XXV ( $1^{\circ}$ semestre 1908), pp. 253-271], $\ 5$.

2) Nella classica Memoria: On the Effect of the Internal Friction of Fluids on the Motion of Pendulums [Transactions of the Cambridge Philosophical Society, Vol. IX (1850), pp. 8-106] [Mathematical and Physical Papers (Cambridge, At the University Press), Vol. III (1901), pp. I-I4I].

3) Suppongo nota al lettore la teoria dei vettori, quale si trova esposta dai sigg. C. BuRali-ForTI e R. Marcolongo, negli ottimi Elementi di Calcolo vettoriale con numerose applicaz̧ioni alla Geometria, alla Meccanica e alla Fisica-matematica (Bologna, Zanichelli, 1909) e le teorie cosi eleganti, suggestive 


\section{I. - Equazioni generali.}

1. Sia $\sigma$ una superficie chiusa, che limita uno spazio $S$, che supponiamo occupato da un liquido incompressibile, viscoso, di densità eguale ad uno, il quale bagni la parete $\sigma$.

Indicando con $\boldsymbol{v}$ il vettore che rappresenta la velocità di una particella generica $P$ di liquido, con $f$ il vettore della forza di massa agente su detta particella, e con $p$ la intensitd della pressione, le equazioni indefinite del moto del liquido sono ${ }^{4}$ )

$$
\begin{aligned}
& \left.k \boldsymbol{\Delta}^{\prime} \boldsymbol{v}=\operatorname{grad} p-\boldsymbol{f}+\frac{d \boldsymbol{v}}{d t}{ }^{5}\right), \\
& \operatorname{div} \boldsymbol{v}=0 \text {, }
\end{aligned}
$$

ove $k$ è il coefficiente di viscosita, e $\frac{d \boldsymbol{v}}{d t}$ indica la derivata totale di $\boldsymbol{v}$ (ottenuta cioè tenendo conto che $\boldsymbol{v}$ contiene esplicitamente $t$, e che inoltre $v$ dipende poi ancora da $t$ pel tramite di $P)$. Scrivendo: $\boldsymbol{v}=\boldsymbol{v}(P, t)$ si ha dunque:

$$
\frac{d v}{d t}=\frac{\partial v}{\partial t}+\frac{d v}{d P} \frac{d P}{d t}=\frac{\partial v}{\partial t}+\frac{d v}{d P} v
$$

ove $\frac{d v}{d P}$ indica l'omografia vettoriale che applicata al vettore $d P$, produce il vettore $d v^{6}$ ).

Nel caso di moti lenti, cioè tali che si possano trascurare i quadrati ed i prodotti

e originali contenute nel recentissimo volumetto degli stessi Autori: Omografie vettoriali con applicazioni alle derivate rispetto ad un punto e alla Fisica-matematica (Torino, Petrini, 1909); nelle citazioni indicherò per brevità questi due libri risp. con (C. v.) e (O. v.). Seguo naturalmente le notazioni vettoriali da essi proposte, le quali, da un'accurata ed ampia analisi dal punto di vista storico, scientifico, logico e pratico, che essi hanno fatto \{cfr.: Per l'unificazione delle notazioni vettoriali (Note I.V) [Rendiconti del Circolo Matematico di Palermo, t. XXIII ( $\mathrm{I}^{\circ}$ sem. 1907), pp. 324-328; t. XXIV (2 ${ }^{\circ}$ sem. 1907), pp. 65-80, 318-332; t. XXV ( ${ }^{\circ}$ sem. 1908), pp. 352-372; t. XXVI ( $2^{\circ}$ sem. 1908), pp. $\left.369-377\right]$, risultano essere le più razionali ed opportune. $\mathrm{E}$ ben giustamente tali notazioni sono già state adottate da molti Autori.

4) Queste equazioni si trovano scritte, coll'algoritmo cartesiano, ad es. nella interessante opera: M. BRILlouin, Leçons sur la viscosité des liquides et des gaz (Paris, Gauthier-Villars, 1907), parte $\mathrm{I}^{\mathrm{a}}$, cap. II, ove sono stabilite collo stesso algoritmo; esse però possono pure ottenersi più semplicemente, e in modo autonomo, con procedimento simile a quello che, per le equazioni dell'Elasticità, è esposto in (O. v.), cap. III.

5) L'operatore $\Delta^{\prime}$, che si applica a vettori, ha per valore $\Delta^{\prime}=$ grad div - rot rot, (O. v., pag. 6I) e la sua espressione in assi cartesiani ortogonali è :

$$
\Delta^{\prime}=\frac{\partial^{2}}{\partial x^{2}}+\frac{\partial^{2}}{\partial y^{2}}+\frac{\partial^{2}}{\partial z^{2}}
$$

$\left.{ }^{6}\right)$ Se $u, v$, w sono le coordinate di $v$ rispetto ad una terna di assi ortogonali, ed $x, y, z$ sono quelle di $P$, si ha:

$$
\frac{d v}{d P} v=\left(u \frac{\partial}{\partial x}+v \frac{\partial}{\partial y}+w \frac{\partial}{\partial z}\right) v
$$


di $\boldsymbol{v}$ e sue derivate, la (I), come mostra l'eguaglianza precedente, assume la forma più semplice:

$$
k \Delta^{\prime} \boldsymbol{v}=\operatorname{grad} p-\boldsymbol{f}+\frac{\partial \boldsymbol{v}}{\partial t} .
$$

Da questa, prendendo la div di ambo i membri, si ha, ricordando la (2):

$$
\operatorname{div} \operatorname{grad} p=\operatorname{div} f
$$

il primo membro è il parametro differenziale secondo di $p$, e indicandolo con $\Delta p^{7}$ ), avremo:
(4)$$
\Delta p=\operatorname{div} f .
$$

Se il moto che si considera è inoltre stazionario, la $\boldsymbol{v}$ non dipende esplicitamente dal tempo, onde la (3) si riduce a:

$$
k \Delta^{\prime} \boldsymbol{v}=\operatorname{grad} p-\boldsymbol{f} .
$$

Se poi le forze ammettono un potenziale $U$ (cioè : $f=\operatorname{grad} U$ ), dalla (4) risulta che la funzione $p-U$ è armonica, e la $(5)$ diventa:

$$
k \boldsymbol{\Delta}^{\prime} \boldsymbol{v}=\operatorname{grad}(p-U) .
$$

Giova notare che quest'equazione si puó immaginare ottenuta dalla $(5)$ stessa, sostituendovi $p$ con $p-U$, e ritenendovi nulla $f$.

Poichè il liquido bagna la parete e ha luogo l'aderenza completa, è chiaro che la condizione al contorno si ottiene scrivendo che la velocita del liquido deve essere eguale a quella della parete con cui è a contatto, dunque:

$$
\boldsymbol{v}=\boldsymbol{v}_{\mathrm{o}} \quad(\mathrm{su} \sigma)
$$

essendo $\boldsymbol{v}_{\mathrm{o}}$ un vettore conosciuto (variabile al variare di $P$ sopra $\sigma$ ). Tale vettore, in forza della (2), e del teorema della divergenza [(C. v.), pag. 95], deve soddisfare alla condizione $\left.{ }^{8}\right)$ :

$$
\int_{\sigma} v_{\mathrm{o}} \times N d \sigma=\mathrm{o}
$$

ove $\boldsymbol{N}$ è un vettore unitario normale alla superficie $\sigma$.

2. In ciò che segue, ci occuperemo solo di moti lenti e stazionari, cioè dell'integrazione delle (5), (2), (7).

E facile vedere intanto che l'integrazione delle $(5),(2),(7)$ si puo sempre ricondurre a quella di equazioni analoghe alle (6), (2), (7), cioè al caso in cui il liquido è soggetto a forze di massa derivanti da un potenziale.

Infatti, per un noto teorema di CLEBSCH, è sempre possibile determinare un numero $U$, ed un vettore $\boldsymbol{u}$, in guisa che :

$$
f=\operatorname{grad} U+\operatorname{rot} \boldsymbol{u}
$$

7) L'operatore $\Delta$, che si applica a numeri, ha l'identica espressione cartesiana di $\Delta^{\prime}$; peró, siccome gli operatori $\Delta, \Delta^{\prime}$ hanno campi di variabilità del tutto differenti, giova indicarli, come é fatto in (O.v.), pag. 6r, con due simboli diversi.

8) Nel citato lavoro del KorN ${ }^{\mathrm{I}}$ ), non è fatto cenno alcuno di questa condizione necessaria. 
allora se determiniamo un vettore $\boldsymbol{v}_{1}$ tale che:

e poniamo:

$$
k \Delta^{\prime} \boldsymbol{v}_{\mathrm{I}}=-\operatorname{rot} \boldsymbol{u}, \operatorname{div} \boldsymbol{v}_{\mathrm{I}}=0 \text {, }
$$

$$
\boldsymbol{v}=\boldsymbol{v}_{\mathrm{r}}+\boldsymbol{w}
$$

ove $\boldsymbol{w}$ è un vettore da determinarsi, le ( $(5),(2),(7)$ diventano:

$$
\begin{gathered}
k \Delta^{\prime} \boldsymbol{w}=\operatorname{grad}(p-U), \operatorname{div} \boldsymbol{w}=0 \quad(\text { in } S), \\
\boldsymbol{w}=\boldsymbol{v}_{\mathrm{o}}-\boldsymbol{v}_{\mathrm{t}} \quad(\mathrm{su} \sigma),
\end{gathered}
$$

le quali dimostrano quanto abbiamo enunciato.

Come vettore $v_{1}$ si puó assumere ad es. l'espressione:

$$
v_{\mathrm{r}}=\frac{\mathrm{I}}{4 \pi k} \operatorname{rot} \int_{S} \frac{u d S}{r},
$$

ove $r$ indica la distanza del punto $P$ dal punto variabile d'integrazione $M$.

Questo teorema di riduzione, che è assai utile nei casi generali, non produce molta semplificazione nel problema speciale che tratteremo. Operiamo percio direttamente sulle (5), (7); indichiamo con $p^{\prime}$ la funzione armonica in $S$ e che su $\sigma$ coincide con $p$, e poniamo :

(9)

allora si ha dalla (4):

e la ( 5 ) diventa:

$$
p=p^{\prime}+F,
$$

$\left(s^{\prime}\right)$

$$
\left\{\begin{array}{cl}
\Delta F=\operatorname{div} f & \text { (in } S) \\
F=0 & \text { (su } \sigma)
\end{array}\right.
$$

ove, ricordiamo, la funzione incognita $p^{\prime}$ è armonica.

3. Il problema definito dalle (5), (2), (7) può dirsi problema idrodinamico interno. Peró spesso è più interessante il problema idrodinamico esterno, cioè la determinazione del moto stazionario lento di un liquido viscoso, che occupa lo spazio indefinito $S$ esterno a $\sigma$, il quale moto si puó immaginare provocato dal moto di un solido in seno ad una massa liquida indefinita. Le equazioni di tale problema sono ancora le $(5),(2)$, (7), ove si aggiunga la condizione che all'infinito il liquido deve rimanere in quiete, cioè che il vettore $\boldsymbol{v}$ si annulli all'infinito.

$\mathrm{La}$ condizione (8), per il vettore arbitrariamente dato $\boldsymbol{v}_{\mathrm{o}}$, non è più necessaria.

L'azione complessiva esercitata dalla massa liquida sul corpo mobile, è caratterizzata da due vettori $\boldsymbol{T}, \boldsymbol{M}$, che sono rispettivamente la risultante e il momento risultante (rispetto ad un punto qualunque) del sistema di pressioni che il liquido esercita sui singoli elementi $d \sigma$ della superficie $\sigma$.

Per il calcolo di $\boldsymbol{T}$ e di $\boldsymbol{M}$ bisogna considerare, oltre l'omografia $\alpha$ delle velocità, espressa da $\alpha=\frac{d v}{d P}$, l'omografia $\beta$ delle pressioni, che è legata alla $\alpha$ dalla relazione:

$$
\left.\beta=p-2 k \mathrm{D} \alpha^{9}\right) \text {, }
$$

9) In quest'unica relazione sono compendiate le 6 equazioni che esprimono le relazioni fra le componenti speciali di pressione e le componenti di deformazione, e che si trovano ad es. nel cap. II della citata opera del Brillouin 4). 
che puó ancora scriversi:

$$
\beta=p-k \frac{d v}{d P}-k \mathrm{~K} \frac{d \boldsymbol{v}}{d P} .
$$

La resistenza $\boldsymbol{T}$ e il momento $\boldsymbol{M}$ vengono allora espressi da:

$$
\begin{aligned}
\boldsymbol{T} & =\int_{\sigma} \beta \boldsymbol{N} d \sigma, \\
\boldsymbol{M} & =\int_{\sigma}(P-O) \wedge \beta \boldsymbol{N} d \sigma,
\end{aligned}
$$

ove $O$ è un punto fisso qualsiasi, ed $\boldsymbol{N}$ è un vettore unitario parallelo alla normale a $\sigma$ e diretto verso l'interno del liquido.

Calcoliamo dapprima $\boldsymbol{T}$; si puó scrivere:

$$
\boldsymbol{T}=\int_{\sigma} p \boldsymbol{N} d \sigma-k \int_{\sigma} \frac{d \boldsymbol{v}}{d P} \boldsymbol{N} d \sigma-k \int_{\sigma} \mathrm{K} \frac{d \boldsymbol{v}}{d P} \boldsymbol{N} d \sigma ;
$$

se ora il vettore $\boldsymbol{v}_{\mathrm{o}}$, dato su $\sigma$, è tale che risulti:

$$
\int_{\sigma} \mathrm{K} \frac{d \boldsymbol{v}}{d P} \boldsymbol{N} d \sigma=\mathrm{o}
$$

la precedente espressione si riduce a:

$$
\boldsymbol{T}=\int_{\sigma} p \boldsymbol{N} d \sigma-k \int_{\sigma} \frac{d \boldsymbol{v}}{d P} \boldsymbol{N} d \sigma,
$$

e poichè $\frac{d \boldsymbol{v}}{d P} \boldsymbol{N}$ non è altro che l'ordinaria derivata di $\boldsymbol{v}$ secondo la normale interna, che possiamo, secondo l'uso, indicare con $\frac{d \boldsymbol{v}}{d n}$, avremo ancora:

La (14) è certo verificata se

$$
\boldsymbol{T}=\int_{\sigma} p \boldsymbol{N} d \sigma-k \int_{\sigma} \frac{d \boldsymbol{v}}{d n} d \sigma .
$$

$$
\lim _{\rho=\infty}\left(\rho^{2} \frac{d v}{d P}\right)=0 \quad(\rho=O P),
$$

cioè se le derivate parziali di $\boldsymbol{v}$ sono, all'infinito, infinitesime d'ordine maggiore di 2; infatti consideriamo una superficie sferica $\omega$ di raggio $\rho_{o}$ molto grande, in guisa che $\sigma$ sia tutta interna ad $\omega$; allora nello spazio $S^{\prime}$ compreso tra $\sigma$ ed $\omega$, si ha: $\operatorname{div} \boldsymbol{v}=0$, e poichè [(O. v.), pag. 59]

risulta

$$
\operatorname{grad} \mathrm{K} \frac{d \boldsymbol{v}}{d P}=\operatorname{grad} \operatorname{div} \boldsymbol{v}
$$

$$
\int_{S^{\prime}} \operatorname{grad} \mathrm{K} \frac{d \boldsymbol{v}}{d P} d S^{\prime}=0 .
$$

Questo integrale di volume si trasforma immediatamente in un integrale di superficie [(O. v.), pag. 72] e si trova cosi:

$$
\int_{\sigma} \mathrm{K} \frac{d \boldsymbol{v}}{d P} \boldsymbol{N} d \sigma+\int_{\omega} \mathrm{K} \frac{d \boldsymbol{v}}{d P} \boldsymbol{N} d \omega=0
$$


ora, se è soddisfatta la (15), è chiaro che facendo crescere indefinitamente $\rho_{0}$, l'integrale esteso ad $\omega$ tende a zero, quindi vediamo che è verificata la (14).

C. D. D.

Si puó scrivere la (II) in altro modo; consideriamo ancora, come dianzi, la superficie sferica $\omega$, allora si ha $[(\mathrm{O} . \mathrm{v}$.$) , pag. 72]$ :

$$
\int_{\sigma} \beta N d \sigma=-\int_{\omega} \beta \boldsymbol{N} d \omega-\int_{S^{\prime}} \operatorname{grad} \beta d S^{\prime}
$$

ma dalle equazioni stesse del moto stazionario e nell'ipotesi di forze conservative di potenziale $U$, risulta $[(\mathrm{O}, \mathrm{v}$.$) , pag. 72,(2)]$ che $\operatorname{grad} \beta=\operatorname{grad} U$, perció avremo:

ovvero anche:

$$
\boldsymbol{T}=-\int_{S^{\prime}} \operatorname{grad} U d S^{\prime}-\int_{\omega} \beta \boldsymbol{N} d \omega,
$$

$$
\boldsymbol{T}=\int_{\sigma} U \boldsymbol{N} d \sigma+2 k \int_{\omega} \mathrm{D} \frac{d \boldsymbol{v}}{d P} \boldsymbol{N} d \omega-\int_{\omega}(p-U) \boldsymbol{N} d \omega .
$$

Se la viscosità è cosi tenue da potersi trascurare $(k=0)$, la $(6)$ mostra che $p-U$ è costante, e poichè $\int_{\omega} \boldsymbol{N} d \omega=0$ per ogni superficie chiusa [(C. v.), pag. 95], si ha:

$$
\boldsymbol{T}=\int_{\sigma} U \boldsymbol{N} d \sigma
$$

la quale espressione non rappresenta altro che la spinta idrostatica dovuta al campo di forza agente sul liquido.

Se l'omografia $\beta$ è all'infinito infinitesima d'ordine maggiore di 2 , e il liquido non è soggetto a forze di massa, si ha $\boldsymbol{T}=0$.

Veniamo al calcolo di $\boldsymbol{M}$. Osservando che [(O. v.), pag. 56]:

la (I2) si può scrivere:

$$
2 D_{\alpha}=2 \frac{d v}{d P}-(\operatorname{rot} v) \wedge,
$$

(I7) $\boldsymbol{M}=\int_{\sigma} p(P-O) \wedge \boldsymbol{N} d \sigma-2 k \int_{\sigma}(P-O) \wedge \frac{d \boldsymbol{v}}{d n} d \sigma+k \int_{\sigma}(P-O) \wedge[(\operatorname{rot} v) \wedge \boldsymbol{N}] d \sigma$.

Considerando la solita superficie sferica $\omega$, si puó ancora porre $\boldsymbol{M}$ sotto la forma:

$$
\boldsymbol{M}=-\int_{S^{\prime}}(P-O) \wedge \operatorname{grad} \beta d S^{\prime}-\int_{\omega}(P-O) \wedge \beta \boldsymbol{N} d \omega,
$$

cioè, se le forze di massa derivano dal potenziale $U$ :

od anche:

$$
\boldsymbol{M}=-\int_{S^{\prime}}(P-O) \wedge \operatorname{grad} U d S^{\prime}-\int_{\omega}(P-O) \wedge \beta \boldsymbol{N} d \omega,
$$

$\boldsymbol{M}=\int_{\sigma} U(P-O) \wedge \boldsymbol{N} d \sigma+2 k \int_{\omega}(P-O) \wedge \mathrm{D} \frac{d \boldsymbol{v}}{d P} \boldsymbol{N} d \omega-\int_{\omega}(p-U)(P-O) \wedge \boldsymbol{N} d \omega$.

Se l'omografia $\beta$ è all'infinito infinitesima d'ordine maggiore di 3 , e il liquido non è soggetto a forze di massa, si ha $\boldsymbol{M}=0$. 


\section{II. - Sfera mobile.}

4. Risolviamo ora il problema interno, supponendo che $\sigma$ sia una superficie sferica di raggio $R$. Il liquido lo supporremo soggetto a forze di massa, rappresentate dal vettore $f$; però non faremo la riduzione indicata dal teorema del $\mathrm{n}^{0} 2$, perchè nelle formole definitive verrebbe a comparire un numero maggiore di quadrature.

Integriamo dapprima il sistema:

$$
\begin{aligned}
k \Delta^{\prime} \boldsymbol{u} & =\operatorname{grad} p^{\prime} & & (\text { in } S), \\
\boldsymbol{u} & =\boldsymbol{v}_{\mathrm{o}} & & (\text { su } \sigma),
\end{aligned}
$$

perciò chiamiamo $v^{\prime}$ il vettore armonico in $S$ (cioè che ivi soddisfa la $\Delta^{\prime} \boldsymbol{v}^{\prime}=0$ ) e che su $\sigma$ coincide con $\boldsymbol{v}_{0}$, e poniamo:

$$
\boldsymbol{u}=\boldsymbol{v}^{\prime}+\left(\rho^{2}-R^{2}\right) \operatorname{grad} \varphi,
$$

ove $\rho$ è la distanza del punto $P$ dal centro $O$ della sfera, e $\varphi$ è una funzione armonica da determinarsi; in tal modo la condizione in superficie è gid verificata. Inoltre:

$$
\Delta^{\prime} \boldsymbol{u}=6 \operatorname{grad} \varphi+4 \rho \frac{d \operatorname{grad} \varphi}{d \rho}=\frac{\mathrm{I}}{k} \operatorname{grad} p^{\prime},
$$

e siccome si verifica subito che:

avremo:

$$
\rho \frac{d \operatorname{grad} \varphi}{d \rho}=\operatorname{grad}\left(\rho \frac{d \varphi}{d \rho}\right)-\operatorname{grad} \varphi,
$$

$$
2 \operatorname{grad} \varphi+4 \operatorname{grad}\left(\rho \frac{d \varphi}{d \rho}\right)=\frac{1}{k} \operatorname{grad} p^{\prime}
$$

onde, prescindendo da una costante, che non ha influenza:

da cui:

$$
2 \varphi+4 \rho \frac{d \varphi}{d \rho}=\frac{I}{k} p^{\prime},
$$

$$
\varphi=\frac{\mathrm{I}}{4 k} \rho^{-\frac{1}{2}} \int_{0}^{\rho} \rho^{-\frac{1}{2}} p^{\prime} d \rho,
$$

e questa funzione, al pari di $p^{\prime}$, è effettivamente armonica. In tal modo è determinato il vettore $\boldsymbol{u}$.

Ció premesso, se si indica con $\boldsymbol{g}$ il vettore tale che:

$$
\left\{\begin{aligned}
k \Delta^{\prime} \boldsymbol{g} & =\operatorname{grad} F-\boldsymbol{f} & & (\text { in } S), \\
\boldsymbol{g} & =\mathrm{o} & & (\text { su } \sigma),
\end{aligned}\right.
$$

è chiaro che il vettore $\boldsymbol{v}$ che soddisfa alle $\left(\varsigma^{\prime}\right),(7)$ è espresso da: $\boldsymbol{v}=\boldsymbol{u}+\boldsymbol{g}$, onde:

$$
\boldsymbol{v}=\boldsymbol{v}^{\prime}+\boldsymbol{g}+\left(\rho^{2}-R^{2}\right) \operatorname{grad} \varphi \text {. }
$$

Rimane da determinare la funzione $p^{\prime}$. Sostituendo la (20) nella (2) e ricordando la formula che dà la div di un prodotto [(C. v.), pag. 68], si ha:

$$
\left(\operatorname{grad} \rho^{2}\right) \times \operatorname{grad} \varphi=-\operatorname{div}\left(\boldsymbol{v}^{\prime}+\boldsymbol{g}\right),
$$


e poichè $\operatorname{grad} \rho^{2}=2(P-O)$, risulta ancora:

$$
2 \rho \frac{d \varphi}{d \rho}=-\operatorname{div}\left(\boldsymbol{v}^{\prime}+\boldsymbol{g}\right)
$$

il primo membro si annulla nel centro della sfera, bisogna percio verificare che anche il secondo membro vi si annulla. Poichè il secondo membro è (come il primo) funzione armonica, ed è noto che il valore di una funzione armonica nel centro di una sfera è eguale alla media dei suoi valori nellinterno della sfera ${ }^{10}$ ), basta verificare se:

$$
\int_{S} \operatorname{div}\left(\boldsymbol{v}^{\prime}+\boldsymbol{g}\right) d S=\mathrm{o},
$$

cioè, per il teorema della divergenza:

$$
\int_{\sigma}\left(\boldsymbol{v}^{\prime}+\boldsymbol{g}\right) \times \boldsymbol{N} d \sigma=0
$$

poichè $\boldsymbol{g}$ si annulla su $\sigma$, la precedente eguaglianza è vera, a causa della (8).

Dalla (2r) si deduce poi, integrando:

$$
\varphi=-\frac{\mathbf{I}}{2} \int_{0}^{\rho} \frac{\mathbf{I}}{\rho} \operatorname{div}\left(\boldsymbol{v}^{\prime}+\boldsymbol{g}\right) d \rho+c,
$$

ove $c$ è una costante arbitraria, che si puó benissimo supporre nulla.

Sostituendo nella (20) si ha:

$$
\boldsymbol{v}=\boldsymbol{v}^{\prime}+\boldsymbol{g}+\frac{\mathrm{I}}{2}\left(R^{2}-\rho^{2}\right) \operatorname{grad} \int_{0}^{\rho} \frac{\mathrm{I}}{\rho} \operatorname{div}\left(\boldsymbol{v}^{\prime}+\boldsymbol{g}\right) d \rho,
$$

e questa formola risolve la questione proposta.

Per determinare la pressione $p$ conviene ricavare $p^{\prime}$ dalla (18); sostituendo in essa a $\varphi$ il suo valore $\left(2 \mathrm{I}^{\prime}\right)$ si ottiene :

$$
p^{\prime}=-2 k \operatorname{div}\left(\boldsymbol{v}^{\prime}+\boldsymbol{g}\right)-k \int_{0}^{\rho} \frac{\mathrm{I}}{\rho} \operatorname{div}\left(\boldsymbol{v}^{\prime}+\boldsymbol{g}\right) d \rho ;
$$

dopo ciò la (9) fornisce la pressione $p$ (a meno di una costante arbitraria).

5. Troviamo l'espressione esplicita di $\boldsymbol{v}$ e $p$.

Intanto la funzione $F$, che soddisfa alle (ro), è data da:

$$
F=-\frac{\mathrm{I}}{4 \pi} \int_{S} G \operatorname{div} f d S,
$$

ove $G$ è la funzione di Green relativa alla sfera $S$. Analogamente il vettore $g$, che soddisfa alle (I9), è espresso da:

$$
\boldsymbol{g}=-\frac{\mathrm{I}}{4 \pi k} \int_{s} G(\operatorname{grad} F-f) d S
$$

ro) Questa proprietà, assai utile, trovasi, anche sotto forma più generale, nella mia Nota: Jrasformazione di alcune funzioni potenziali [Rendiconti del Circolo Matematico di Palermo, t. XXII ( $2^{\circ}$ sem. 1906), pp. 220-232], pag. 229, linea 17. Essa è stata ritrovata, due anni appresso, da S. ZAREMBa nella Memoria: Sur l'intégration de l'équation biharmonique [Bulletin international de l'Académie des Sciences de Cracovie, Anné I908, pp. 1-29], $\$ 3$. 
ora si ha, per una funzione qualunque $\psi$ :

$$
\int_{S} G \operatorname{grad} \int_{S} G \psi d S=2 \pi \int_{S}(M-P) G \psi d S,
$$

ove $M$ indica il punto variabile d'integrazione; infatti, entrambi i membri si annullano su $\sigma$, e inoltre i loro $\boldsymbol{A}^{\prime}$ sono eguali in $S$, come si verifica inmediatamente. Dunque $\boldsymbol{g}$ puó scriversi, tenendo conto della (23):

Inoltre :

$$
\boldsymbol{g}=\frac{\mathrm{I}}{4 \pi k} \int_{S} G\left[\frac{\mathrm{I}}{2}(M-P) \operatorname{div} \boldsymbol{f}+\boldsymbol{f}\right] d S .
$$

$$
v^{\prime}=\frac{R^{2}-\rho^{2}}{4 \pi R} \int_{\sigma} \frac{v_{0} d \sigma}{r^{3}}
$$

$r$ essendo la distanza del punto $P$ dal punto $M$ variabile su $\sigma$.

Dalla $\left(20^{\prime}\right)$ si trae quindi:

(24)

$$
\left\{\begin{array}{c}
v=\frac{R^{2}-\rho^{2}}{4 \pi R} \int_{\sigma} \frac{v_{0} d s}{r^{3}}+\frac{\mathrm{I}}{4 \pi k} \int_{S} G\left[\frac{\mathrm{I}}{2}(M-P) \operatorname{div} f+f\right] d S \\
\left(+\frac{\mathrm{I}}{8 \pi}\left(R^{2}-\rho^{2}\right) \operatorname{grad} \int_{0}^{\rho} \frac{\mathrm{I}}{\rho} \operatorname{div}\left\{\frac{R^{2}-\rho^{2}}{R} \int_{\sigma} \frac{\boldsymbol{v}_{0} d \sigma}{r^{3}}+\frac{\mathrm{I}}{k} \int_{S} G\left[\frac{\mathrm{I}}{2}(M-P) \operatorname{div} f+f\right] d S\right\} d \rho .\right.
\end{array}\right.
$$

Per la pressione $p$ si ha, per la (9):

$$
\left\{\begin{array}{l}
p=-\frac{\mathrm{I}}{4 \pi} \int_{S} G \operatorname{divf} d S-\frac{k}{2 \pi} \operatorname{div}\left\{\frac{R^{2}-\rho^{2}}{R} \int_{\sigma} \frac{v_{0} d \sigma}{r^{3}}+\frac{\mathrm{I}}{k} \int_{S} G\left[\frac{\mathrm{I}}{2}(M-P) \operatorname{div} f+f\right] d S\right\} \\
-\frac{k}{4 \pi} \int_{0}^{\rho} \frac{\mathrm{I}}{\rho} \operatorname{div}\left\{\frac{R^{2}-\rho^{2}}{R} \int_{\sigma} \frac{v_{0} d \sigma}{r^{3}}+\frac{\mathrm{I}}{k} \int_{S} G\left[\frac{\mathrm{I}}{2}(M-P) \operatorname{div} f+f\right] d S\right\} d \rho .
\end{array}\right.
$$

6. Le precedenti formule si semplificano se le forze di massa soddisfano ad ipotesi particolari. Cosi ad es. se supponiamo verificata la condizione

$$
\operatorname{div} \boldsymbol{f}=\mathrm{o},
$$

allora la (4) mostra che la pressione $p$ è funzione armonica, e nelle (24), (25) alcuni termini scompaiono.

Se invece le forze ammettono un potenziale $U$, ricordando l'osservazione fatta a proposito dell'equazione (6), si trae immediatamente dalle (20'), (22):

e dalle (24), (25):

$$
\boldsymbol{v}=\boldsymbol{v}^{\prime}+\frac{\mathrm{I}}{2}\left(R^{2}-\rho^{2}\right) \operatorname{grad} \int_{0}^{\rho} \frac{\mathrm{I}}{\rho} \operatorname{div} \boldsymbol{v}^{\prime} d \rho,
$$$$
\text { (24') } \quad v=\frac{R^{2}-\rho^{2}}{4 \pi R} \int_{\sigma} \frac{v_{0} d \sigma}{r^{3}}+\frac{R^{2}-\rho^{2}}{8 \pi R} \operatorname{grad} \int_{0}^{\rho} \frac{\mathrm{I}}{\rho} \operatorname{div}\left[\left(R^{2}-\rho^{2}\right) \int_{\sigma} \frac{v_{0} d \sigma}{r^{3}}\right] d \rho
$$$$
\left(25^{\prime}\right) p-U=-\frac{k}{2 \pi} \operatorname{div}\left[\left(R^{2}-\rho^{2}\right) \int_{\sigma} \frac{u_{\mu} d \sigma}{r^{3}}\right]-\frac{k}{4 \pi R} \int_{0}^{\rho} \frac{\mathrm{I}}{\rho} \operatorname{div}\left[\left(R^{2}-\rho^{2}\right) \int_{\sigma} \frac{\boldsymbol{v}_{0} d \sigma}{r^{3}}\right] d \rho \text {. }
$$

Rend. Circ. Matem. Palermo, t. XXX (20 senr. 19ro). - Stampato il 20 aprile 19 ro. 
La formula $\left(24^{\prime}\right)$ puó ancora scriversi sotto la forma seguente ${ }^{\text {II }}$ ):

$$
v=\frac{R^{2}-\rho^{2}}{4 \pi R} \int_{\sigma}\left[\frac{v_{0}}{r^{3}}+\frac{\mathrm{I}}{2 \rho} \int_{0}^{\rho} \operatorname{grad} \operatorname{div}\left(v_{0} \frac{R^{2}-\rho^{2}}{r^{3}}\right) d \rho\right] d \sigma,
$$

la quale, oltre che mostrare che l'integrale rispetto a $\rho$ si puó calcolare sotto forma finita, permette di stabilire, con procedimento analogo a quello che si adopera per le formule che danno la deformazione di una sfera elastica isotropa ${ }^{\mathrm{r}}$ ), che - subordinatamente alla condizione (8) - la continuità di $v_{\mathrm{o}}$ è sufficiente perchè le $\left(24^{\prime}\right),\left(25^{\prime}\right)$ risolvano effettivamente la questione proposta.

Nel citato lavoro del Korn è supposto invece che le componenti di $v_{0}$ abbiano derivate prime continue, soddisfacenti inoltre ad una particolare condizione.

7. Il problema esterno si risolve con procedimento perfettamente analogo; supponendo, per brevità, che le forze di massa ammettano un potenziale $U$, allora le formule risolutive sono:

$$
\begin{aligned}
& v=v^{\prime}+\frac{I}{2}\left(R^{2}-\rho^{2}\right) \operatorname{grad} \int_{\infty}^{\rho} \frac{\mathrm{I}}{\rho} \operatorname{div} v^{\prime} d \rho, \\
& p-U=-2 k \operatorname{div} \boldsymbol{v}^{\prime}-k \int_{\infty}^{\rho} \frac{\mathrm{I}}{\rho} \operatorname{div} \boldsymbol{v}^{\prime} d \rho,
\end{aligned}
$$

che si possono dedurre dalle $\left(\mathbf{2 0}^{\prime \prime}\right),\left(\mathbf{2 2}^{\prime}\right)$ cambiando il limite inferiore zero degli integrali (rispetto a $\rho$ ), in $\infty$. In esse $\boldsymbol{v}^{\prime}$ è espresso da:

$$
v^{\prime}=\frac{\rho^{2}-R^{2}}{4 \pi R} \int_{\sigma} \frac{v_{0} d \sigma}{r^{3}}
$$

perció sostituendo si hanno formule del tutto simili alle $\left(24^{\prime}\right),\left(25^{\prime}\right)$, la prima delle quali si puó poi mettere sotto la forma $\left(24^{\prime \prime}\right)$, donde si deduce che la continuità di $v_{0}$ è sufficiente perchè le (26), (27) risolvano effettivamente il problema proposto.

II) In virtù della formula:

$$
\operatorname{grad}\left[\rho^{-n} \int_{0}^{\rho} \rho^{n-1} \psi d \rho\right]=\rho^{-n-1} \int_{0}^{\rho} \rho^{n} \operatorname{grad} \psi d \rho,
$$

ove $\psi$ è funzione armonica, ed $n$ costante positiva o nulla.

12) LaURICELla, Sulle formole che dànno la deformazione di una sfera elastica isotropa [Rendiconti della R. Accademia dei Lincei, vol. XIII, $2^{\circ}$ semestre I904, pp. $583-590$ ]. Il ragionamento del LAURICELLA è fatto sulle espressioni in coordinate cartesiane delle formule risolutive, però è facilissimo ridurlo a forma assoluta. $\mathrm{La}\left(24^{\prime \prime}\right)$ porge le seguenti formule in coordinate cartesiane:

$$
\begin{aligned}
u=\frac{R^{2}-\rho^{2}}{4 \pi R}\left\{\int_{\sigma} u_{0}\left[\frac{\mathrm{I}}{r^{3}}+\frac{\mathrm{I}}{2 \rho} \int_{0}^{\rho} \frac{d^{2}}{d x^{2}}\left(\frac{R^{2}-\rho^{2}}{r^{3}}\right) d \rho\right] d \sigma\right. \\
\left.+\int_{\sigma} v_{0}\left[\frac{\mathrm{I}}{2 \rho} \int_{0}^{\rho} \frac{d^{2}}{d x d y}\left(\frac{R^{2}-\rho^{2}}{r^{3}}\right) d \rho\right] d \sigma+\int_{\sigma} w_{0}\left[\frac{1}{2 \rho} \int_{0}^{\rho} \frac{d^{2}}{d x d \chi}\left(\frac{R^{2}-\rho^{2}}{r^{3}}\right) d \rho\right] d \sigma\right\},
\end{aligned}
$$

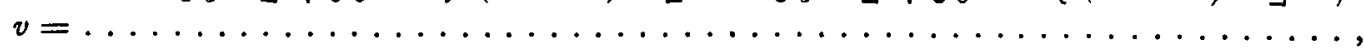

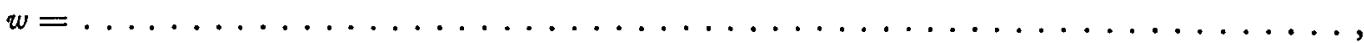

ove $u, v, w$ sono le coordinate di $v$ rispetto ad una terna di assi ortogonali, e $u_{a}, v_{0}, w_{0}$ quelle di $\boldsymbol{v}_{\mathrm{o}}$. Apparisce di qui l'immensa semplicità e brevità della (24") in confronto delle precedenti espressioni cartesiane. 
La resistenza $\boldsymbol{T}$ che la sfera incontra muovendosi nel liquido viscoso, è data dalla (I3); peró, senza fare alcuna ipotesi sul vettore dato $\boldsymbol{v}_{0}$, si puó dimostrare che è verificata la (I4), cioè che la (I3) si riduce alla ( $\mathrm{I}^{\prime}$ ).

Infatti, dalla (26) si ha:

$$
\mathrm{K} \frac{d \boldsymbol{v}}{d \bar{P}} \boldsymbol{N}=\mathrm{K} \frac{d \boldsymbol{v}^{\prime}}{d \bar{P}} \boldsymbol{N}+\frac{\mathrm{I}}{2} \mathrm{~K} \frac{d}{d P}\left[\left(R^{2}-\rho^{2}\right) \operatorname{grad} \int_{\infty}^{\rho} \frac{\mathrm{I}}{\rho} \operatorname{div} \boldsymbol{v}^{\prime} d \rho\right] \boldsymbol{N} ;
$$

l'ultimo termine si trasforma facilmente mediante una nota formula [(O. v.), pag. 53] e si trova cosi che vale (ricordando che per i punti di $\sigma$ si ha $p=R$ ):

$$
\frac{\mathrm{I}}{2}\left(\boldsymbol{N} \times \operatorname{grad} \int_{\infty}^{\rho} \frac{\mathrm{I}}{\rho} \operatorname{div} \boldsymbol{v}^{\prime} d \rho\right) \operatorname{grad}\left(R^{2}-\rho^{2}\right)=\frac{\mathrm{I}}{2}\left(\frac{\mathrm{I}}{\rho} \operatorname{div} \boldsymbol{v}^{\prime}\right) \operatorname{grad}\left(R^{2}-\rho^{2}\right)=-\left(\operatorname{div} \boldsymbol{v}^{\prime}\right) \boldsymbol{N},
$$

perciò, introducendo la funzione $\mathrm{C}$ [(O. v.), nota di pag. 60 e pag. Ior $]$ :

Ora si puó porre:

$$
\mathrm{K} \frac{d \boldsymbol{v}}{d \vec{P}} \boldsymbol{N}=-\mathrm{KC} \frac{d \boldsymbol{v}^{\prime}}{d P} \boldsymbol{N} .
$$

$$
v^{\prime}=\frac{a}{\rho}+v^{\prime \prime}
$$

ove $\boldsymbol{a}$ è un vettore costante, e $\boldsymbol{v}^{\prime \prime}$ un vettore (armonico) che all'infinito diventa infinitesimo come $\frac{\mathrm{I}}{\rho^{2}}$; e poichè si verifica subito che

$$
\mathrm{KC} \frac{d\left(\frac{a}{p}\right)}{d P} \boldsymbol{N}=\mathrm{o},
$$

risulta:

$$
\mathrm{K} \frac{d \boldsymbol{v}}{d P} \boldsymbol{N}=-\mathrm{KC} \frac{d \boldsymbol{v}^{\prime \prime}}{d P} \boldsymbol{N} .
$$

Ora, il secondo membro, e quindi anche il primo, è, all'infinito, infinitesimo d'ordine maggiore di 2 , perciò ricordando la (I6), ne segue la (I4).

8. Trattiamo, come primo esempio di problema esterno, un caso particolare assai notevole; quello in cui la sfera si muove di moto traslatorio (lento) in un liquido soggetto a forze conservative, il suo centro descrivendo una retta $O x$, con velocita costante $\boldsymbol{v}_{\mathrm{o}}$.

Immaginiamo di imprimere a sfera e liquido la velocitd opposta a quella della sfera, cioè la velocità $-\boldsymbol{v}_{0}$; allora è chiaro che con ciò non viene alterato lo stato di moto relativo della sfera rispetto al liquido, $m a$ il nuovo stato di moto (in cui la sfera $\mathrm{e}$ in riposo) è evidentemente stazionatio, e per esso valgono le equazioni indefinite (6), (2), colle condizioni ai limiti:

Ponendo:

$$
v=\mathrm{o} \quad(\mathrm{su} \sigma) ; \quad \boldsymbol{v}=-\boldsymbol{v}_{\mathrm{o}} \quad \text { (all'infinito). }
$$

$$
\boldsymbol{w}=\boldsymbol{v}+\boldsymbol{v}_{\mathrm{o}},
$$

è chiaro che $\boldsymbol{w}$ rappresenta la velocita delle particelle liquide nel moto stazionario provocato dalla sfera nel liquido originariamente in riposo, e si hanno per $\boldsymbol{w}$ ancora le 
equazioni indefinite (6), (2), colle condizioni ai limiti:

$$
\boldsymbol{w}=\boldsymbol{v}_{\mathrm{o}}(\mathrm{su} \sigma) ; \quad \boldsymbol{w}=\mathrm{o} \quad \text { (all'infinito) }
$$

La determinazione di $\boldsymbol{w}$ si effettua per mezzo delle (26), (27); si ha in questo caso:

onde :

$$
\boldsymbol{v}^{\prime}=\frac{R}{\rho} \boldsymbol{v}_{\mathrm{o}}
$$

e successivamente:

$$
\operatorname{div} v^{\prime}=\left(\operatorname{grad} \frac{R}{\rho}\right) \times v_{\mathrm{o}}
$$

$$
\begin{aligned}
\int_{\infty}^{\rho} \frac{\mathrm{I}}{\rho} \operatorname{div} \boldsymbol{v}^{\prime} d_{\rho} & =v_{0} \times \int_{\infty}^{\rho} \frac{\mathrm{I}}{\rho} \operatorname{grad} \frac{R}{\rho} d \rho=v_{0} \times \operatorname{grad}\left(\rho \int_{\infty}^{\rho} \frac{R}{\rho^{3}} d \rho\right) \\
& =R v_{0} \times \operatorname{grad} \frac{-\mathrm{I}}{2 \rho}=\frac{R}{2 \rho^{3}} \boldsymbol{v}_{0} \times(P-O),
\end{aligned}
$$

quindi :

$$
\operatorname{grad} \int_{\infty}^{\rho} \frac{\mathrm{I}}{\rho} \operatorname{div} \boldsymbol{v}^{\prime} d \rho=-\frac{3 R}{2 \rho^{5}}\left[v_{\mathrm{o}} \times(P-O)\right](P-O)+\frac{R}{2 \rho^{3}} \boldsymbol{v}_{\mathrm{o}} ;
$$

onde le (26), (27) forniscono:

$$
\begin{aligned}
\boldsymbol{w} & =\frac{R}{4 \rho^{3}}\left(3 p^{2}+R^{2}\right) \boldsymbol{v}_{\mathrm{o}}+\frac{3 R\left(p^{2}-R^{2}\right)}{4 p^{5}}\left[\boldsymbol{v}_{\mathrm{o}} \times(P-O)\right](P-O), \\
p & =U+\frac{3^{k R}}{2 p^{3}} \boldsymbol{v}_{\mathrm{o}} \times(P-O) .
\end{aligned}
$$

Supponendo $\rho$ costante, vediamo come varia $\boldsymbol{w}$, al variare dell'angolo $\theta$ che il vettore $P-O$ fa con $v_{\mathrm{o}}$. La (28) puó scriversi:

ove:

$$
\boldsymbol{w}=a \boldsymbol{v}_{\mathrm{o}}+b \cos \theta(P-O)
$$

$$
a=\frac{R\left(3 \rho^{2}+R^{2}\right)}{4 \rho^{3}}, \quad b=\frac{3 R\left(\rho^{2}-R^{2}\right)}{4 \rho^{4}} \bmod v_{0},
$$

perció il vettore $\boldsymbol{w}$ è la somma di due altri, di cui l'uno è parallelo a $\boldsymbol{v}_{\mathrm{o}}$, e l'altro che è parallelo a $(P-O)$, ha una lunghezza eguale alla corda della circonferenza di diametro $b \rho$, inclinata di $\theta$ sul diametro passante per un estremo di essa. La costruzione grafica di $\boldsymbol{w}$ è dunque immediata.

L'angolo $\varphi$ che la velocità $\boldsymbol{w}$ fa con $\boldsymbol{v}_{\mathrm{o}}$ è in conseguenza sempre minore di un retto, e il suo massimo valore $p_{0}$ è determinato, come si verifica subito, dalla condizione :

$$
\operatorname{sen} \varphi_{0}=\frac{3\left(\rho^{2}-R^{2}\right)}{9 p^{2}-R^{2}}
$$

L'angolo $\theta_{0}$ a cui corrisponde questa massima inclinazione $\varphi_{0}$, è determinato da:

$$
\theta_{0}=\frac{\pi}{4}+\frac{\varphi_{0}}{2} \text {. }
$$

I due angoli $\varphi_{0}$ e $\theta_{0}$ sono dunque indipendenti dalla velocita $v_{0}$.

Si possono ottenere con facilita le linee di corrente, le quali giacciono su piani pas- 
santi per la retta $O x$ : la loro equazione differenziale è infatti:

$$
\boldsymbol{w} \wedge d P=0
$$

e chiamando $r$ la distanza del punto $P$ dalla retta $O x, \mathrm{e} w_{r}$, $w_{\rho}$ le lunghezze delle proiezioni (ortogonali) di $\boldsymbol{w}$ risp. sulla perpendicolare ad $O x$, e sopra $P-O$, l'equazione precedente porge facilmente:

ora, dalla (28) segue:

$$
w_{r} d p-w_{\rho} d r=0 ;
$$

$$
\begin{aligned}
& w_{r}=\frac{3 R\left(\rho^{2}-R^{2}\right)}{4 \rho^{5}} r v_{0} \times(P-O) \\
& w_{\rho}=\frac{\boldsymbol{w} \times(P-O)}{\rho}=\frac{2 R\left(3 \rho^{2}-R^{2}\right)}{4 \rho^{4}} \boldsymbol{v}_{0} \times(P-O)
\end{aligned}
$$

quindi :

$$
\frac{3\left(\rho^{2}-R^{2}\right)}{\rho\left(3 \rho^{2}-R^{2}\right)} d \rho=\frac{2 d r}{r}
$$

ed integrando si ha come equazione delle linee di corrente:

$$
\frac{\left(3 p^{2}-R^{2}\right) r^{2}}{p^{3}}=\text { costante. }
$$

La funzione di corrente di Stokes è quindi data da:

$$
\left.\psi=\lambda \frac{\left(3 \rho^{2}-R^{2}\right) r^{2}}{\rho^{3}}{ }^{\mathrm{r} 3}\right)
$$

ove $\lambda$ è un fattore costante, che si trova facilmente essere eguale ad $R \bmod \frac{\boldsymbol{v}_{\mathrm{o}}}{4}$.

Per calcolare la resistenza $\boldsymbol{T}$, applichiamo la $\left(13^{\prime}\right)$; si ha intanto dalla (28), nei punti di $\sigma$ :

$$
\frac{d \boldsymbol{v}}{d p}=-\frac{3 \boldsymbol{v}_{\mathrm{o}}}{2 \mathrm{R}}+\frac{3}{2 R^{3}}\left[\boldsymbol{v}_{\mathrm{o}} \times(P-O)\right](P-O),
$$

ovvero, per la (29):

quindi la ( $\left.13^{\prime}\right)$ porge:

$$
\frac{d \boldsymbol{v}}{d p}=-\frac{3 \boldsymbol{v}_{o}}{2 R}+\frac{p-U}{k} \boldsymbol{N}
$$

$$
\boldsymbol{T}=6 \pi k R \boldsymbol{v}_{\mathrm{o}}+\int_{\sigma} U \boldsymbol{N d} \sigma .
$$

Se il liquido non è soggetto a forze di massa, $\boldsymbol{T}$ si riduce al primo termine, e si ritrova cosi una nota formola di Stokes ${ }^{\mathbf{1 4}}$ ). Da essa egli ha dedotto una conseguenza interessante, col ragionamento seguente: se la sfera di raggio $R$ e densita $n$, discende (verticalmente) in un liquido viscoso di densità $\mu$, sotto l'azione della gravità, la forza che agisce sul solido ha per intensita:

$$
\frac{4}{3} \pi R^{3} g(n-\mu)
$$

х) Loc. cit. $\left.{ }^{2}\right)$, pag. 56.

I4) Loc. cit. ${ }^{2}$ ), pag. 59 . 
se perció la sfera cade dal riposo, la sua velocità non puó crescere indefinitamente, ma deve tendere ad un limite $V$, che è determinato dall'equazione:

da cui :

$$
\frac{4}{3} \pi R^{3} g(n-\mu)=6 \pi k R V,
$$

$$
\left.V=\frac{2}{9} g R^{2} \frac{\eta-\mu}{k}{ }^{15}\right) \text {. }
$$

Questa formula è stata applicata recentemente alla determinazione della carica di un elettrone $\left.{ }^{16}\right)$.

Per calcolare il momento $\boldsymbol{M}$ rispetto al centro $O$ della sfera, applichiamo la (I7), il cui primo termine (nel caso della sfera) è identicamente nullo; la rot del secondo termine della (28) è nulla per i punti di $\sigma$, e si ha poi subito:

$$
\operatorname{rot} v=-\frac{3}{2 R} N \wedge v_{0},(\text { su } \sigma),
$$

donde segue:

risultato prevedibile.

$$
\boldsymbol{M}=\mathrm{o} \text {, }
$$

9. Come secondo esempio supponiamo che la sfera $\sigma$ ruoti uniformemente, con velocità angolare costante $\omega$, intorno a un suo diametro; si ha allora:

$$
v_{o}=\omega \Omega \wedge(P-O),
$$

essendo $\boldsymbol{\Omega}$ un vettore unitario e parallelo all'asse di rotazione.

$\mathrm{Ne}$ risulta:

$$
\boldsymbol{v}^{\prime}=\frac{R^{3}}{\rho^{3}} \omega \Omega \wedge(P-O),
$$

che puó pure scriversi [(C. v.), pag. 68]:

$$
\boldsymbol{v}^{\prime}=R^{3} \omega\left(\operatorname{grad} \frac{\mathrm{I}}{\rho}\right) \wedge \boldsymbol{\Omega}=R^{3} \omega \operatorname{rot} \frac{\mathbf{\Omega}}{\rho},
$$

da cui apparisce che div $\boldsymbol{v}^{\prime}=0$.

Le (26), (27) si riducono percio ad:

$$
\boldsymbol{v}=\boldsymbol{v}^{\prime}, \quad p=U .
$$

Le linee di corrente sono determinate dall'equazione differenziale:

cioè, sostituendo:

$$
v \wedge d P=o,
$$

$$
(\boldsymbol{\Omega} \times d P)(P-O)-[(P-O) \times d P] \mathbf{\Omega}=0,
$$

dunque si deve avere:

ed integrando:

$$
\mathbf{\Omega} \times d P=0, \quad(P-O) \times d P=0,
$$

$$
\mathbf{\Omega} \times(P-O)=\text { cost. }, \quad(P-O)^{2}=\text { cost. }
$$

I5) Loc. cit. ${ }^{2}$ ), pag. 60.

16) Cfr. ad es. Battelli-Occhialini-Chella, La Radioattività (Bari, Gius. Laterza e figli, 1909), $\$ 22$. 
le quali dicono che le linee di corrente sono le intersezioni di piani normali all'asse di rotazione, con sfere concentriche a $\sigma$; cosa evidente.

La resistenza $\boldsymbol{T}$ si puó calcolare mediante la (13"); si ha:

$$
\frac{d \boldsymbol{v}}{d \rho}=-\frac{R^{3}}{\rho^{3}} 2 \omega \Omega \wedge \boldsymbol{N},
$$

perciò l'omografia $\frac{d \boldsymbol{v}}{d P}$ è, all'infinito, infinitesima di $3^{\circ}$ ordine, quindi la ( $\left.13^{\prime \prime}\right)$ si riduce a:

risultato prevedibile.

$$
\boldsymbol{T}=\int_{\sigma} U \boldsymbol{N} d \sigma
$$

Il momento $\boldsymbol{M}$ si ottiene dalla (17), e poichè si trova facilmente:

si conclude:

$$
(\operatorname{rot} v) \wedge N=-\frac{R^{3}}{\rho^{3}} \omega \Omega \wedge N
$$

$$
\boldsymbol{M}=3 k R \omega \int_{\sigma} \boldsymbol{N} \wedge(\boldsymbol{\Omega} \wedge \boldsymbol{N}) d \sigma
$$

cioè, sviluppando [(C. v.), pag. 33]:

$$
\begin{aligned}
& \boldsymbol{M}=3 k R \omega\left\{4 \pi R^{2} \boldsymbol{\Omega}-\frac{\mathrm{I}}{R} \int_{\sigma}[(P-O) \times \Omega] \boldsymbol{N} d \sigma\right\}, \\
& \boldsymbol{M}=3 k R \omega\left[4 \pi R^{2}-\frac{\mathrm{I}}{R} \frac{4}{3} \pi R^{3}\right] \boldsymbol{\Omega}=8 \pi k R^{3} \omega \mathbf{\Omega} .
\end{aligned}
$$

Se il liquido, anzichè essere indefinitamente esteso, fosse limitato da una superficie sferica $\sigma_{\mathrm{I}}$, di raggio $R_{1}$, concentrica a $\sigma$, e rotante con velocita costante $\omega_{1}$ intorno allo stesso diametro di $\sigma$, si avrebbe:

$$
\boldsymbol{v}=a \mathbf{\Omega} \wedge(P-O)+\frac{b}{\rho^{3}} \mathbf{\Omega} \wedge(P-O),
$$

ove $a, b$ sono costanti, determinate dalle equazioni:

$$
a+\frac{b}{R^{3}}=\omega, \quad a+\frac{b}{R_{\mathrm{I}}^{3}}=\omega_{1} .
$$

Come altro esempio supponiamo, più in generale, che la sfera ruoti uniformemente, con velocità angolare costante $\omega$, intorno ad un asse situato a distanza $a$ dal suo centro. Rispetto ad un osservatore rigidamente connesso colla sfera, il moto è evidentemente stazionario, e la questione si riduce a determinare $\boldsymbol{v}$, sapendo che

$$
\boldsymbol{v}_{\mathrm{o}}=\omega \mathbf{\Omega} \wedge(P-A),
$$

ove $A$ è la proiezione ortogonale del centro $O$ sull'asse di rotazione; si puó ancora scrivere:

$$
\boldsymbol{v}_{\mathrm{o}}=\omega \Omega \wedge(P-O)+\omega a \boldsymbol{I},
$$

ove $\boldsymbol{I}$ è un vettore unitario, normale al piano passante per l'asse e pel centro $O$.

Il valore di $\boldsymbol{v}$ si puó dedurre dalle (30), (28). 


\section{III. - Piano indefinito.}

Io. Consideriamo un piano $\sigma$ (indefinito) situato in una massa liquida illimitata, e vediamo qual'è il moto stazionario lento provocato in tale massa dal movimento di $\sigma$. Supponiamo che le forze di massa ammettano un potenziale $U$, e chiamiamo $z$ un asse normale a $\sigma$ in un punto $A$, e fissiamo su esso il verso positivo; basterà evidentemente esaminare il moto stazionario del liquido situato nella regione positiva dell'asse $z$.

La velocità $\boldsymbol{v}$ della particella liquida situata nel punto $P$, si può determinare con procedimento simile a quello adoperato precedentemente nel caso della sfera. Più semplicemente, possiamo ricavarla, mediante un passaggio al limite, dalla formula trovata nel caso della sfera, analogamente a quanto si puó fare nella teoria della elasticità ${ }^{\mathbf{1 7}}$ ).

Supponiamo che la sfera di raggio $R$, considerata nel $\mathrm{n}^{\circ} 7$, sia tangente al piano $\sigma$ nel punto $A(z=0)$, ed abbia il suo centro $O$ nel punto $z=-R$. Allora, supposto che il punto $P$ sia al finito, e chiamando $z$ la sua distanza dal piano $\sigma(z>0)$, si ha al limite, per $R=\infty$ :

$$
\begin{gathered}
\lim \frac{\rho}{R}=\mathrm{I} ; \quad \lim \frac{\rho^{2}-(R+z)^{2}}{R}=0, \quad \text { onde } \lim \frac{\rho^{2}-R^{2}}{R}=2 z ; \\
\lim \frac{\rho}{R} \frac{d f}{d \rho}=\lim \frac{P-O}{R} \times \operatorname{grad} f=I \times \operatorname{grad} f=\frac{d f}{d z},
\end{gathered}
$$

ove $\boldsymbol{I}$ è un vettore unitario, normale a $\sigma$ e avente per verso quello dell'asse $z$.

Inoltre, dicendo $\sigma^{\prime}$ la superficie della sfera considerata:

$$
\begin{aligned}
\frac{\rho}{R} \frac{d}{d \rho} \int_{\infty}^{\rho} \frac{1}{\rho} \operatorname{div}\left(R v^{\prime}\right) d \rho & =\operatorname{div} \boldsymbol{v}^{\prime}=\operatorname{div}\left(\frac{\rho^{2}-R^{2}}{4 \pi R} \int_{\sigma^{\prime}} \frac{\boldsymbol{v}_{0} d \sigma^{\prime}}{r^{3}}\right) \\
& =\frac{\rho^{2}-R^{2}}{4 \pi R} \operatorname{div} \int_{\sigma^{\prime}} \frac{\boldsymbol{v}_{0} d \sigma^{\prime}}{r^{3}}+\frac{1}{2 \pi} \frac{P-O}{R} \times \int_{\sigma^{\prime}} \frac{\boldsymbol{v}_{0} d \sigma^{\prime}}{r^{3}} ;
\end{aligned}
$$

perciò :

$$
\begin{aligned}
\lim \frac{d}{d z} \int_{\infty}^{\rho} \frac{\mathrm{I}}{\rho} \operatorname{div}\left(R \boldsymbol{v}^{\prime}\right) d \rho & =\frac{z}{2 \pi} \operatorname{div} \int_{\sigma} \frac{\boldsymbol{v}_{\mathrm{o}} d \sigma}{r^{3}}+\frac{\mathrm{I}}{2 \pi} \boldsymbol{I} \times \int_{\sigma} \frac{\boldsymbol{v}_{\mathrm{o}} d \sigma}{r^{3}} \\
& =\frac{\mathrm{I}}{2 \pi} \operatorname{div}\left(z \int_{\sigma} \frac{\boldsymbol{v}_{\mathrm{o}} d \sigma}{r^{3}}\right)=-\frac{\mathrm{I}}{2 \pi} \frac{d}{d z} \operatorname{div} \int_{\sigma} \frac{\boldsymbol{v}_{\mathrm{o}} d \sigma}{r},
\end{aligned}
$$

sicchè si puó ritenere:

$$
\lim \int_{\infty}^{\rho} \frac{I}{\rho} \operatorname{div}\left(R \boldsymbol{v}^{\prime}\right) d \rho=-\frac{\mathbf{I}}{2 \pi} \operatorname{div} \int_{\sigma} \frac{\boldsymbol{v}_{\mathrm{o}} d \sigma}{r},
$$

17) Cfr. Tedone, Saggio di una teoria generale delle equazioni dellequilibrio elastico per un corpo isotropo [Annali di Matematica pura ed applicata, serie III, tomo VIII (1903), pp. I29-I80], cap. V. 
onde dalle (26), (27) si deduce :

$$
\begin{gathered}
\left.v=-\frac{\mathrm{I}}{2 \pi} \frac{d}{d z} \int_{\sigma} \frac{v_{\mathrm{o}} d \sigma}{r}+\frac{z}{2 \pi} \operatorname{grad} \operatorname{div} \int_{\sigma} \frac{\boldsymbol{v}_{\mathrm{o}} d \sigma}{r}{ }^{\mathrm{s}}\right), \\
p=U+\frac{k}{\pi} \frac{d}{d z} \operatorname{div} \int_{\sigma} \frac{v_{\mathrm{o}} d \sigma}{r},
\end{gathered}
$$

e queste formule risolvono la questione proposta.

Introducendo la derivata secondo la normale interna, esse possono ancora scriversi sotto la forma seguente:

$$
\begin{gathered}
v=\frac{\mathrm{I}}{2 \pi} \int_{\sigma} \boldsymbol{v}_{\mathrm{o}} \frac{d \frac{\mathrm{I}}{r}}{d n} d \sigma-\frac{\mathrm{I}}{4 \pi} \int_{\sigma} \frac{d r^{2}}{d n} \operatorname{grad} \operatorname{div} \frac{\boldsymbol{v}_{\mathrm{o}}}{r} d \sigma, \\
p=U-\frac{k}{\pi} \operatorname{div} \int_{\sigma} \frac{d \frac{\mathrm{I}}{r}}{\boldsymbol{v}_{\mathrm{o}}} \frac{r}{d n} d \sigma .
\end{gathered}
$$

Poichè le forze di massa sono conservative, nell'espressione di $\boldsymbol{v}$ non comparisce mai il coefficiente di- viscositd.

Torino, ottobre 1909.

Tommaso Boggio.

${ }^{18}$ ) $\mathrm{Si}$ suppone che, in questo passaggio al limite, l'integrale $\int_{\sigma} \frac{v_{\mathrm{o}} d \sigma}{r}$ resti finito anche al limite. 